\title{
BMJ Open Effect of milk protein content in Toddler formula on later BMI and obesity risk: protocol of the multicentre randomised controlled Toddler Milk Intervention (ToMI) trial
}

\author{
Veit Grote (D) , ${ }^{1}$ Vanessa Jaeger, ${ }^{1}$ Joaquin Escribano, ${ }^{2,3}$ Marta Zaragoza, ${ }^{2,4}$ \\ Mariona Gispert, ${ }^{2}$ Dominik Grathwohl, ${ }^{5}$ Berthold Koletzko ${ }^{1}$
}

To cite: Grote V, Jaeger V, Escribano J, et al. Effect of milk protein content in Toddler formula on later BMI and obesity risk: protocol of the multicentre randomised controlled Toddler Milk Intervention (ToMl) trial. BMJ Open 2021;11:e048290. doi:10.1136/ bmjopen-2020-048290

- Prepublication history and additional supplemental material for this paper are available online. To view these files, please visit the journal online (http://dx.doi.org/10.1136/ bmjopen-2020-048290).

Received 23 December 2020 Accepted 21 November 2021

\section{ABSTRACT}

Introduction Reduction of milk protein content in infant formula provided during the first year of life has been shown to reduce early weight gain and obesity later in life. While rapid weight gain during the first 2 years of life is one of the strongest early predictors of obesity, the role of animal protein intake beyond the first year of life is unclear. The aim of this study is to examine the role of milk protein during the second year of life in healthy children on weight gain and obesity risk in preschool age.

Methods and analysis This randomised, double-blinded study enrolled 1618 children aged 11.5-13.5 months in Spain and Germany into two groups receiving isocaloric toddler milk with differing protein content during the second year of life. The experimental formula contains $1.5 \mathrm{~g} / 100 \mathrm{kcal}$ and the control formula $6.15 \mathrm{~g} / 100 \mathrm{kcal}$ protein and otherwise equal formula composition, except for modified fat content to achieve equal energy density. The primary endpoint is body mass index (BMI)-for-age z-score at the age of 24 months adjusted for BMl at 12 months of age. The children are followed until 6 years of age.

Ethics and dissemination Ethics approval was obtained from the ethical committees of the LMU University Hospital Munich, Germany (Nr. 555-15) and at Institut d'Investigació Sanitaria Pere Virgili, Reus, Spain (Ref. CEIm IISPV 013/2016). We aim at publishing results in peer-reviewed journals and sharing of results with study participants. Trial registration number NCT02907502.

\section{INTRODUCTION}

A randomised double blind controlled clinical trial demonstrated that reducing protein intake in infant formula provided in the first year of life lowers early weight gain until 2 years of age. ${ }^{1}$ Data from the same study (CHildhood Obesity Project (CHOP) trial) demonstrated that lower protein supply with formula fed in the first year of life also markedly reduced body mass index (BMI) and obesity risk at school age. ${ }^{2}$ The results of the CHOP trial contributed to enhanced
Strengths and limitations of this study

- This study uses a randomised and double blinded design to minimise potential confounding and biases.

- The multicentre design of this study with sites in Spain and Germany increases external validity of study results.

- The follow-up of the cohort is planned until 6 years of age and will provide the possibility to examine long-term effects of the intervention.

- Conclusions will be limited to effects of dairy protein provided with milk based drinks in the second year of life and cannot be extrapolated to effects of total dietary protein supply.

promotion of breast feeding and efforts in reducing the protein content in infant and follow-on formula. ${ }^{34}$

It remains unclear which child age period is most sensitive to a modified protein intake, and whether limiting protein intake during the second year of life would also achieve benefits for prevention of excessive weight gain and later obesity. Observational studies find a consistent association of later overweight and obesity with total protein intake and in particular of milk protein intake, not only during infancy but also during the preschool age. ${ }^{5-9}$ A systematic review on the effects of dietary protein intake concluded that the first 2 years of life are the most sensitive time period. ${ }^{10}$

The untoward programming effect of a high early protein intake on later obesity risk has been linked to its effects on increasing plasma and tissue concentrations of insulinogenic amino acids, insulin and insulinlike growth factor 1 (IGF-1), which appear to induce a higher weight gain during the first 2 
years of life as well as an enhanced adipogenic activity. ${ }^{11}$ Such effects of an infant formula higher protein content on insulinogenic amino acids, insulin and IGF-1 levels have been shown in the double-blind randomised CHOP trial. ${ }^{12-14}$

Milk protein seems to play a key role in growth regulation during early childhood. Protein intake is the main contributor for nutritional regulation of the IGF-I axis. ${ }^{1516}$ Milk protein enhances serum IGF-1 to a greater extent than meat protein. ${ }^{17}$ This might explain the more pronounced effect of milk protein compared with other proteins on the later risk of obesity that has been reported. ${ }^{8}$

Average protein intake of young children in Europe and other regions is much higher than metabolic requirements. During the second year of life, $30 \%-50 \%$ of total daily protein is comprised of dairy products, ${ }^{18} 19$ indicating particular opportunities to reduce overall protein consumption though modifying dairy protein intake.

Therefore, we designed a randomised controlled trial to examine the role of milk protein intake during the second year of life on child growth and later obesity risk. If a reduction of milk protein during the second year of life has an appreciable effect on growth and obesity development, respective dietary modification may be translated into the practice of toddler feeding.

\section{Main objective}

We aim at evaluating the effect of two iso-energetic milk products for young children with differing protein content on growth during the second year of life.

\section{Secondary study objectives}

Besides treating the study as an intervention study as described in detail below, the study incorporates a longer follow-up and is also considered a cohort study. Data obtained and produced should be scientifically exploited for explorative analysis specifically addressing the interplay and factors that influence child feeding, growth and development, physical activity, metabolism and disease prevention.

\section{METHODS AND ANALYSIS \\ Study design and population}

The Toddler Milk Intervention trial (ToMI trial) is designed as a two-arm, parallel, randomised, double blind controlled trial to evaluate toddler milk products with different protein content. The study is conducted at university hospitals in Munich, Germany and in Tarragona and Reus, Spain.

The target population are healthy children at the age of 1 year. The children are enrolled if they meet the inclusion and exclusion criteria outlined in table 1.

\section{Intervention-formula composition}

Two investigational formulas are used. Both formulas are based on cow's milk. The protein is unmodified from cow's milk and has the same casein:whey protein ratio in both formulas. The experimental formula contains $0.72 \mathrm{~g}$ protein $/ 100 \mathrm{~mL}(1.5 \mathrm{~g} / 100 \mathrm{kcal})$, with a protein content that is similar to breast milk in advanced lactation. The control formula contains $2.95 \mathrm{~g}$ protein $/ 100 \mathrm{~mL}$ $(6.15 \mathrm{~g} / 100 \mathrm{kcal})$ which is comparable to standard cows' milk with $2 \%$ fat content. Contents of energy, carbohydrates, vitamins and minerals are very similar for both formulas (table 2). In order to reach the same energy content in both formulas, the fat content varies between experimental $(4.25 \mathrm{~g}$ fat $/ 100 \mathrm{kcal})$ and control formula $(2.16 \mathrm{~g}$ fat $/ 100 \mathrm{kcal})$ but the lipid composition and the ratio of milk fat/vegetable oils is the same. Both formulas were developed and produced by the sponsor for this trial and were not tested in any other studies before the trial.

Table 1 Inclusion and exclusion criteria of the Toddler Milk Intervention trial

Inclusion criteria Exclusion criteria

Legal guardians signed the written informed consent.

Infant who is breastfed at least twice in 24 hours at time of enrolment.

Child was born full term ( $\geq 37+0$ weeks of gestation). Infant who usually does not drink $300 \mathrm{~mL}$ of cow's milk and/or formula milk per day.

Child's birth weight is between 2.5 and $4.5 \mathrm{~kg}$. Cow's milk allergy.

Child is born from a singleton pregnancy. Lactose intolerance.

Child's age at enrolment is between 11.5 and Institutionalised children.

\section{5 months.}

Child's legal guardians are of legal age and they have sufficient local language skills to understand the study information, informed consent and study procedure.

\section{Child and child's parents are willing to fulfil the Children who participated in any other interventional clinical trial 4 requirements of the study protocol and procedures. weeks prior to enrolment.}

Diagnosed disorder, which interfere with nutrition or growth (eg, coeliac disease, inflammatory bowel disease).

Child's family is available via phone or e-mail throughout the whole study. 
Table 2 Nutritional composition of the interventional products (toddler milks) that are based on cow's milk with the same casein:whey protein ratio

\begin{tabular}{|ccc}
\hline & $\begin{array}{l}\text { Experimental } \\
\text { toddler milk } \\
\text { (as prepared, per } \\
\text { 100 mL) }\end{array}$ & $\begin{array}{l}\text { Control toddler } \\
\text { milk } \\
\text { (as prepared, } \\
\text { per 100 mL) }\end{array}$ \\
\hline Energy & $201 \mathrm{~kJ} / 48 \mathrm{kcal}$ & $201 \mathrm{~kJ} / 48 \mathrm{kcal}$ \\
\hline Protein & $0.72 \mathrm{~g}$ & $2.95 \mathrm{~g}$ \\
\hline Fat & $2.0 \mathrm{~g}$ & $1.0 \mathrm{~g}$ \\
\hline $\begin{array}{l}\text { Saturated fatty } \\
\text { acids }\end{array}$ & $0.8 \mathrm{~g}$ & $0.4 \mathrm{~g}$ \\
\hline Carbohydrates & $6.7 \mathrm{~g}$ & $6.7 \mathrm{~g}$ \\
\hline Lactose & $6.7 \mathrm{~g}$ & $6.6 \mathrm{~g}$ \\
\hline Other & $<0.1 \mathrm{~g}$ & $<0.1 \mathrm{~g}$ \\
\hline Salt & $0.1 \mathrm{~g}$ & $0.1 \mathrm{~g}$ \\
\hline Vitamines & & $66 \mu \mathrm{g}$ \\
\hline Vitamin A & $71 \mu \mathrm{g}$ & $1.3 \mu \mathrm{g}$ \\
\hline Vitamine D & $1.2 \mu \mathrm{g}$ & $14.2 \mu \mathrm{g}$ \\
\hline Folic acid & $14.9 \mu \mathrm{g}$ & $0.2 \mu \mathrm{g}$ \\
\hline Vitamin B12 & $0.2 \mu \mathrm{g}$ & $6.9 \mathrm{mg}$ \\
\hline Vitamin C & $6.4 \mathrm{mg}$ & \\
\hline Minerals & & $115 \mathrm{mg}$ \\
\hline Calcium & $115 \mathrm{mg}$ & $0.5 \mathrm{mg}$ \\
\hline Micronutrients & & $0.6 \mathrm{mg}$ \\
\hline Iron & $0.5 \mathrm{mg}$ & \\
\hline Zinc & $0.3 \mathrm{mg}$ & \\
\hline
\end{tabular}

Dose, route of administration and schedule of formula

Participating families receive the formula as milk powder (one can comprises about $400 \mathrm{~g}$ of product) and are advised to prepare the formula according to the instructions which were identical for all product codes. It is recommended to consume at least $300 \mathrm{~mL}$ of formula per day. Further, parents are encouraged to substitute with the study formula any milk intake from the child's diet. The intake of other dairy products such as cheese or yoghurt is accepted.

The intervention starts with the first study visit at around 1 year of age and ends with the third study visit at around 2 years of age. The study formula is given to the parents at no costs and is delivered directly to subject's home. Subject's compliance is regularly checked by telephone and personal interviews. After the end of the intervention, return and pick-up of remaining cans is organised. If not possible, families are advised to destroy remaining infant formula cans.

\section{Discontinuation criteria}

Discontinuation of the trial can be either due to withdrawal of consent at any time or due to the investigator's decision that continuation within the trial might impair child's health. All efforts will be undertaken to follow children irrespective of their study product consumption with all planned assessments.

\section{Primary endpoint}

The primary endpoint is BMI-for-age z-score (based on the WHO Multicentre Growth Reference Study ${ }^{20}$ ) at the age of 24 months adjusted for BMI-for-age z-score at 12 months of age.

\section{Secondary objectives and endpoints}

The secondary objectives serve to evaluate the safety and efficacy of the two milk products used and to complement the primary endpoint. Secondary endpoints will also be adjusted for baseline measurements if available. Secondary endpoints are:

- BMI-for-age z-score at 72 months.

- The percentage of overweight and obese children at 24 months of age according to the US Centers for Disease control and Prevention (CDC) definition: overweight is at and above the 85th to less than 95th percentile and obese 95 th percentile or greater.

- The percentage of overweight and obese children at 72 months of age.

- Anthropometric measures (z-scores for weight, length and head, waist and arm circumference at 12, 18, 24, 48 and 72 months of age; hip circumference at 48 and 72 month of age).

- Subcutaneous fat distribution (from skinfold thickness at 24, 48 and 72 months of age).

- Total body fat and lean mass (from BodPod measurements at 24, 48 and 72 months of age).

- Blood pressure (48 and 72 month of age).

- Child development (24 and 48 months of age).

- Metabolic and endocrine markers (IGF-1, IGF-Binding Protein (BP)2, IGF-BP3, insulin, leptin, adiponectin, ghrelin, lipid profile and complete blood count at 12 , 24 and 72 month of age).

- Serum albumin, urea, creatinine, amino acids at 12, 24, 72 months of age and ferritin and 25-OH-vitamin $\mathrm{D}$ (at 24 months of age).

- Metabolic profile (from plasma at 12, 24 and 72 months of age and from urine samples at 12, 18, 24, 48 and 72 months of age).

- Urine markers (calcium, C-peptide, creatinine urea nitrogen at 12, 18, 24, 48 and 72 months of age).

Furthermore, the following hypotheses will be examined:

- Total energy intake is not affected by the low protein formula.

- Total protein intake is lower in the group of protein reduced formula.

- Plasma concentrations of essential amino acids and of IGF-1 at the age of 24 months are lower in the low protein formula group compared with the high protein formula group.

- Systolic and diastolic blood pressure measurements at the ages of 48 and 72 months are lower in the 
low protein formula group compared with the high protein formula group.

- Body fat mass at age 24 months is lower in the low protein formula group compared with the high protein formula group.

- DNA methylation affects the association of protein intake and BMI.

- Protein intake affects DNA methylation.

- DNA methylation affects the association of protein intake and the metabolic profile.

DNA methylation is currently only planned as an option provided additional funding can be secured.

\section{Sample size}

The sample size calculation is based on the observations from the CHOP-study. ${ }^{1}$ This trial examined the difference in BMI-for-age z-scores between two groups of children fed a higher or lower protein content formula during the first year of life. At 24 months of age the BMI for age z-score difference between both formula groups was $0.2 \mathrm{SD}$. The absolute difference in protein content between intervention and control group in the CHOPtrial was lower (Infant formula: $0.8 \mathrm{~g} / 100 \mathrm{~mL}$; Follow-on formula: $1.6 \mathrm{~g} / 100 \mathrm{~mL}$ ) compared with the ToMI-trial $(2.2 \mathrm{~g} / 100 \mathrm{~mL})$. Despite a higher protein difference, we expect a lower effect of the intervention due to the lower contribution of milk to the total protein intake in the second year of life. Thus, we assume a slightly lower mean difference in BMI for age z-score of $0.15 \mathrm{SD}$ at 24 months of life.

The sample size was calculated with an anticipated effect size on BMI for age z-score of $0.15 \mathrm{SD}$ and a SD of 0.9 . Assuming a power of $80 \%$ and a significance level of $5 \%$ (two-sided alpha of 0.05 ), a sample size of 566 subjects per intervention arm is calculated. Therefore, 1132 subjects in total are needed. To have enough power to detect also a difference of the same magnitude at 72 months (6 years) of age, at an assumed loss to follow-up of $30 \%$, a final sample size of 1618 subjects was estimated.

\section{Recruitment}

The study sites in Munich, Reus and Tarragona followed somewhat different recruitment strategies due to different local conditions. In Germany all inhabitants are registered in central registries. The public registries provided the study team for this defined research on a regular basis addresses of all families with children in the required age group (about 26000 per year). These families living in Munich and about 70 surrounding municipalities were contacted once by postal mail and invited to contact the study team if interested in participation in the trial.

In Spain two recruitment strategies were used for both sites covering about 3000 births per year. First, telephone contacts from families who delivered their child at either of the two hospitals were available. These families were contacted directly. Second, recruitment interviews at primary healthcare centres were conducted. In these primary healthcare centres, Spanish children are seen for healthcare examinations and for vaccinations.

\section{Allocation of study formula and blinding}

The study formula cans are labelled with one of eight codes. Four codes each are assigned to the intervention or the control group, respectively. The allocation of the codes is performed online by study staff after check of inclusion and exclusion criteria within the data capture tool (iMedidata, Medidata Balance, New York, USA) using balanced randomisation stratified by country. After enrolment of the subject into the trial, study staff dispense the assigned study formula to the study participant along with instructions for formula preparation.

The study is double blinded with all persons involved in local organisation and conduct of the study such as study staff, principal investigator, project manager, biostatistician, data manager, trial monitor and laboratory analysts being unaware of the code allocation. After the code break for the primary outcome analysis, subjects will receive a new identification id in the analysis data to hamper the unblinding for above persons in the further follow-up. An emergency code break by an Investigator may be requested only in case of an unexpected serious adverse event (SAE) suspected to be related to the investigational product.

\section{Data collection and management}

During the intervention period three visits at the hospital are scheduled at 12, 18 and 24 months of age (figure 1). At baseline socioeconomic data and data on health, growth and nutrition by 24-hours recalls during the first year of life are assessed. At each visit anthropometric measurements are performed and urine samples are collected. Blood is taken at 12 and 24 months of age. Additionally, at 24 months of age body composition using an air displacement plethysmography (BodPod COSMED, Rome, Italy) as well as physical activity measurement using an accelerometer device (Actigraph wGT3X-BT, Pensacola, Florida, USA) is performed. Further, data of child's development based on parent answers of the Ages \& Stages questionnaire (ASQ-3, Brookes Publishing Co., Inc., USA) are collected.

For follow-up, two additional visits are scheduled at 48 and 72 months of age with anthropometric, body composition and physical activity measurements and collection of urine samples and food frequency questionnaires (Eating Habits Questionnaire). ${ }^{21}$ Furthermore, socioeconomic data and data on health are updated and data on nutrition behaviour are collected. At 48 months of age, the ASQ-3 is used again. Blood is taken at 72 months of age.

The main primary aim of the nutritional assessment during the intervention phase is to see if the intervention groups differ in nutritional intake. Therefore, a 24-hour recall is used. While the second year of life is still considered a nutritional transition period, nutrition patterns are more stable between 48 and 72 months of age and 
Telephone interview

Nutrition
D Developmental test

Anthropometry / Questionnaires
Urine \& Blood (12, 24 ,72 months)

Physical Activity \& Body Composition

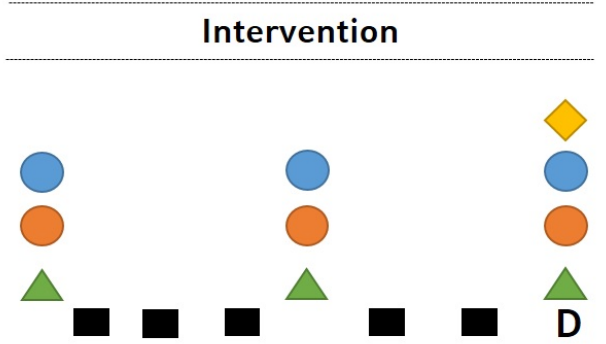

Higher protein toddler milk

Randomization
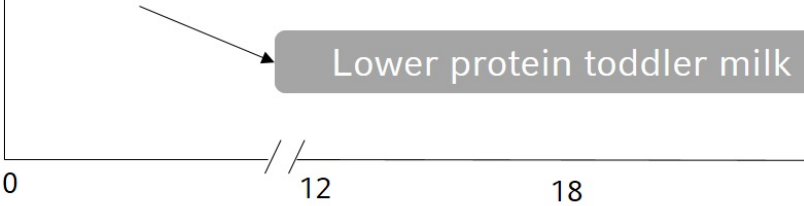

$-/ 1$

218

24

36

48

Months of age

Figure 1 Assessments in children participating in the Toddler Milk Intervention trial.

analysis of food patterns are more relevant. Therefore, a Food Frequency Questionnaire (FFQ) is used for the later time points.

During all study visits and at several additional telephone calls between visits, parents are asked for health problems (including adverse events (AEs)) and compliance. For compliance the intake of study milk and any discontinuation of study milk intake with reasons are determined. The number of consumed cans will be used to determine the average study milk consumption.

Collected data are organised in different databases. To organise and document all contacts with study participants and to coordinate the shipment of the study product, a web-based participant management tool is used (developed jointly with MedSciNet AB, Stockholm, Sweden). In this database, personal data are saved and stored on a secured data server. This database is separated from the other databases which store all medical, nutritional and laboratory data.

All collected health data are primarily captured on paper except data from questionnaires on physical activity and food frequency questionnaires that are entered by families using LimeSurvey (LimeSurvey GmbH, Hamburg, Germany). All other data are transferred from paper into web-based databases. Nutritional data from 24-hours recalls are entered into Nutritics (NUTRITICS Ltd, Dublin, Ireland) with nutritional information from the German nutritional database Bundeslebensmittelschluessel (BLS) 3.02 and complemented with the nutritional composition from a variety of commercial infant foods and local foods, obtained directly from the label, producer websites or local food composition databases. All other data are entered into iMedidata (New York, USA).

Laboratory samples are processed according to a laboratory SOP. In general, aliquots have 2-D barcodes, are scanned, linked with the subject ID and stored into 96-well racks at $-80^{\circ} \mathrm{C}$ for later analysis. Only blood count, lipid status and $\mathrm{HbA1c}$ are measured locally on the day of blood sampling.

To ensure data quality, study staff is trained in regular intervals, and procedures are harmonised among study centres by regular contact and monitoring. Furthermore, anthropometric measurements are performed at least twice and data entry is strictly checked for consistency and plausibility by the monitor. Standard operating procedures for all measurements are in place; anthropometric measurements are based on the WHO Growth Standards study. $^{20}$

\section{Statistical methods}

A statistical analysis plan is created before final code break for the analysis of primary and secondary outcomes. For the statistical analysis, the full analysis dataset (FAS) and the per-protocol-dataset (PP) will be considered. The FAS comprises all randomised subjects who consumed at least one can of investigational product and was considered reasonable and as close as possible to the intention to treat (ITT) ideal as we dealt with a healthy population that participated not for treatment reasons. The PP compromises all subjects included in the FAS and that were compliant with the aimed product consumption (mean consumption of the recommended daily minimum amount of investigational product of 
$300 \mathrm{~mL} / \mathrm{d}$ ). Compliance will be primarily assessed by the number of tins used by the study subject. A Blind Data Review Meeting with participants of the sponsor and the investigators will define specific rules and definitions for lack of compliance. No imputation of missing values is foreseen.

The primary endpoint will be analysed in the FAS by linear regression (analysis of covariance, ANCOVA) and corrected for BMI-for-age z-score at baseline, study centre and gender. The results of the final model will be compared with further adjusted models and analysis in the PP group; possible effect modification of the primary outcome will be also considered.

Secondary analyses supporting primary objective:

1. BMI-for-age z-score at 72 months.

2. The percentage of overweight and obese children at 24 months of age according to CDC definition: Overweight at and above the 85 th to less than 95 th percentile and obese 95 th percentile or greater.

3. The percentage of overweight and obese children at 72 months of age.

In order to control the experiment wise false positive rate, the listed hierarchy (primary-secondary endpoints) will be maintained in interpreting these outcomes. The incidence of overweight and obese children at 24 and 72 months of age shall be also estimated according to International Obesity Task Force definition. ${ }^{22}$ The percentage of overweight and obese children will be analysed by the method of Sauzet $e t a l^{23}$

Secondary endpoints include anthropometric measures, dietary and biochemical data. We will use z-scores of WHO growth standards for anthropometry measures at months 12, 18, 24 and 48. We will use a likelihood-ratio test to examine if there is a longitudinal treatment effect. Additionally, treatment differences at each visit will be analysed using ANCOVA. The ANCOVA approach was chosen so that treatment differences and $p$ value do not depend on the stage of analysis. A further supportive analysis with a mixed linear model shall be performed at 6 years of age. Fixed effects shall be the intervention group, age, gender and a two-way interaction between child age and intervention group will be included. The random effects shall be a random intercept and slope.

Dietary data is collected by 24-hours recalls or food frequency questionnaires, which allow us to test for differences in macronutrient intake using ANCOVA. Hence, we are able to analyse if subjects change their dietary habits over time.

Biochemical data are often log-normal distributed. In order to analyse this kind of data properly, we will logtransform the data to achieve approximately normal distributed residuals.

\section{Interim analysis}

To ensure safety of the intervention, an interim analysis is planned when 260 subjects have completed the intervention (at 24 months of age). Non-inferiority for weightfor-age z-score has to be shown. This must be the case in both FAS and PP. A non-inferiority boundary for weightfor-age z-score of minus $0.5 \mathrm{SD}$ was chosen according to Onyango et al. ${ }^{24}$ The same model as for the primary analysis is used. To demonstrate non-inferiority, the lower bound of the two-sided $95 \%$ CI of the model based treatment difference must be larger than the non-inferiority margin.

If non-inferiority is shown, the study is continued as planned. Otherwise, a second stage interim analysis is performed including the first 390 subjects who have completed the intervention. Furthermore, the safety evaluation will consider endpoints including AEs, anthropometry, laboratory data and protein intake. Based on the results of the interim analysis and in accordance with the charter of the Data Monitoring Committee (DMC), the DMC will recommend either continuing the study as planned or performing the second stage interim analysis. The DMC is independent and consists of expert clinicians and statisticians with no competing interest. The planned interim safety analysis took place in June 2018 and no safety concerns were detected.

Besides the interim analysis, safety is continuously observed by blinded online monitoring of individual growth curves based on the WHO growth charts. If a considerable number of subjects drop below the median growth curve, an interim analysis will be initiated and the DMC will review unblinded data.

\section{Harms}

Any AEs which lead to an untoward medical occurrence except for diagnostic and therapeutic non-invasive and invasive procedures will be recorded during the entire intervention period until 30 days after last study milk intake. After these 30 days, only AE's which are related to the intervention treatment will be recorded. Each $\mathrm{AE}$ will be rated according to its severity and its relationship to the study milk. Additionally, severe adverse events (SAEs) which for example, require inpatient hospitalization will be reported to the safety manager within 24 hours after notice and will be followed up until the outcome is known. A participant insurance is in place.

\section{Monitoring}

A commercial monitoring company reviews the process, $\mathrm{AE}$ reporting, data capturing and corresponding source data on a regular basis to ensure protocol compliance, accuracy and completeness.

\section{Protocol versions}

Issue date: 15 September 2020; version identifier: 5; number of protocol amendments: 5 ; initial version: 9 March 2016. First modification: 30 March 2016. Besides adaptation from requests of both ethical committees before the start of the study and several minor changes due to misspecifications in the protocol, several clarifications were needed, for example, to provide more clarity and criteria for study termination before regular completion of the study, clarification in the statistical interpretation 
of secondary endpoints, addition of new secondary endpoints physical activity and $\mathrm{HbAlc}$, the adaptation to the new European data protection rules in 2018, and a change in exclusion criteria to allow the inclusion of children that are breastfed once per day. Furthermore, an extensive specification of the safety interim analysis after inclusion of 260 children was added in 2018 and more details for collection of AEs separating the collection into two periods, during and after the intervention, were provided.

\section{Ethical considerations}

This study is conducted in compliance with the International Conference on Harmonization guidelines and the Declaration of Helsinki and complies with Good Clinical Practice guidelines. Ethics approval was obtained from the ethical committees of the university hospitals at the Ludwig-Maximilian University in Munich, Germany (Projekt Nr. 555-15) and at the Institut d'Investigació Sanitaria Pere Virgili, Reus, Spain (Ref. CEIm IISPV 013/2016. All protocol amendments were and will be approved by the ethical committee prior to implementation. All procedures and databases were approved by the local data protection agent and are in line with local and EU general data protection regulations.

Written informed consent is collected by study staff from all legal guardians prior to study inclusion in adherence with regulatory requirements with additional consent for genetic analysis. Each subject receives oral as well as written informed consent in plain language with adequate time in advance to make an informed decision about study participation. The latest informed consent form for both study sites is enclosed in the online supplementary (online supplemental file 1). All participants reconsented for any additional measurement added to the protocol.

\section{Patient and public involvement}

The study protocol was primarily developed at a public university hospital without involvement of the sponsor. There was no further public or patient involvement.

\section{Public dissemination and data availability}

Study results will be published in peer-reviewed journals and presented on national and international conferences. Study results will also be communicated to participants. Results will be written-up and published by the investigators without help of professional writers. Authorship will depend on relevant contribution to the study. Investigators have full research freedom and have full access to all data. The full study protocol will be made available on request. The participant-level dataset is not currently planned to be available because consent was not obtained for the sharing of such data from participant's parents/ legal guardians or the Institutional Ethics Committees.

\section{Trial status and time course of the trial}

The study started to recruit subjects in September 2016 and finished recruitment of 1625 children in October
2019. The intervention phase will last until October 2020. The database closure for the analysis of the primary outcome is planned for the first quarter of 2021. The follow-up will be completed around October 2025.

\section{Funding, role of the sponsor and Investigators}

The sponsor has allocated a fixed budget for each study centre to recruit and follow the subjects. The sponsor is producing the study product and distributes the study product to the study subjects. The sponsor is funding the monitoring of the study. The primary protocol was outlined by the investigators and was jointly further developed by investigators and sponsor. Data management will be primarily done by the sponsor, except parts of the compliance checks, checks of biosamples and body composition data, as well as nutritional and physical activity data. The primary analysis will be performed by the sponsor. The investigators have to approve the statistical analysis plan and will have full access to all the data. Any published interpretation of the data has to be in mutual agreement between sponsor and investigator without hampering the research freedom of the investigators. The urinary metabolic profile will be performed by the sponsor, all other laboratory measurements by the investigators. BK is the coordinating principal investigator, with VG being his deputy, JE is principal investigator in Spain.

\section{Author affiliations}

${ }^{1}$ Dept. of Paediatrics, Dr. von Hauner Children's Hospital, LMU University Hospitals Munich, Munich, Bavaria, Germany

${ }^{2}$ Dept. Paediatrics, Universitat Rovira i Virgili, Tarragona, Spain

${ }^{3}$ Dept. Paediatrics, Hospital Universitari Sant Joan de Reus, Reus, Spain

${ }^{4}$ Dept. Paediatrics, Hospital Universitari de Tarragona Joan XXIII, Tarragona, Spain

${ }^{5}$ Nestle Research Center, Lausanne, Switzerland

Contributors VG and VJ wrote the manuscript. VG and BK provided the original outline of the protocol; JE, MZ, MG, and DG participated in the design and set-up of the study. BK, JE, MZ, MG, and DG critically revised the content of the manuscript.

Funding This study is sponsored by Societé de Produits Nestlé S.A., Switzerland (Avenue Nestlé 55, CH-1800 Vevey)—formerly named Nestec. The work of VG, VJ and BK is/was financially supported in part by the European Commission H2O2O Programme Lifecycle-733206, the European Joint Programming Initiative Project NutriPROGRAM, the German Ministry of Education and Research, Berlin (Grant Nr. 01 Gl 0825), the German Research Council (Ko912/12-1 and INST 409/224-1 FUGG). BK is the Else Kröner Seniorprofessor of Paediatrics at LMU - University of Munich, financially supported by Else Kröner-Fresenius-Foundation, LMU Medical Faculty and LMU University Hospitals.

Competing interests The institutions of VG, VJ, BK, JE, MZ, MG receive funding by the sponsor to conduct the study and DG is employed by the sponsor of the study.

Patient consent for publication Not applicable.

Provenance and peer review Not commissioned; externally peer reviewed.

Supplemental material This content has been supplied by the author(s). It has not been vetted by BMJ Publishing Group Limited (BMJ) and may not have been peer-reviewed. Any opinions or recommendations discussed are solely those of the author(s) and are not endorsed by BMJ. BMJ disclaims all liability and responsibility arising from any reliance placed on the content. Where the content includes any translated material, BMJ does not warrant the accuracy and reliability of the translations (including but not limited to local regulations, clinical guidelines, terminology, drug names and drug dosages), and is not responsible for any error and/or omissions arising from translation and adaptation or otherwise. 
Open access This is an open access article distributed in accordance with the Creative Commons Attribution Non Commercial (CC BY-NC 4.0) license, which permits others to distribute, remix, adapt, build upon this work non-commercially, and license their derivative works on different terms, provided the original work is properly cited, appropriate credit is given, any changes made indicated, and the use is non-commercial. See: http://creativecommons.org/licenses/by-nc/4.0/.

ORCID iD

Veit Grote http://orcid.org/0000-0001-7168-2385

\section{REFERENCES}

1 Koletzko B, von Kries R, Closa R, et al. Lower protein in infant formula is associated with lower weight up to age $2 \mathrm{y}$ : a randomized clinical trial. Am J Clin Nutr 2009;89:1836-45.

2 Weber M, Grote V, Closa-Monasterolo R, et al. Lower protein content in infant formula reduces BMI and obesity risk at school age: followup of a randomized trial. Am J Clin Nutr 2014;99:1041-51.

3 Koletzko B, Demmelmair H, Grote V, et al. Optimized protein intakes in term infants support physiological growth and promote long-term health. Semin Perinatol 2019;43:151153.

4 European Commission. Commission DELEGATED regulation (EU) 2016/128 of 25 September 2015 supplementing regulation (EU) No $609 / 2013$ of the European Parliament and of the Council as regards the specific compositional and information requirements for food for special medical purposes. Official Journal of the European Union 2016:L25/30 http://data.europa.eu/eli/reg_del/2016/128/oj

5 Parizkova J, Rolland-Cachera MF. High proteins early in life as a predisposition for later obesity and further health risks. Nutrition 1997;13:818-9.

6 Karaolis-Danckert N, Günther ALB, Kroke A, et al. How early dietary factors modify the effect of rapid weight gain in infancy on subsequent body-composition development in term children whose birth weight was appropriate for gestational age. Am J Clin Nutr 2007;86:1700-8.

7 Günther ALB, Buyken AE, Kroke A. Protein intake during the period of complementary feeding and early childhood and the association with body mass index and percentage body fat at $7 \mathrm{y}$ of age. Am J Clin Nutr 2007;85:1626-33.

8 Günther ALB, Remer T, Kroke A, et al. Early protein intake and later obesity risk: which protein sources at which time points throughout infancy and childhood are important for body mass index and body fat percentage at 7 y of age? Am J Clin Nutr 2007;86:1765-72.

9 Ohlund I, Hernell O, Hörnell A, et al. BMI at 4 years of age is associated with previous and current protein intake and with paternal BMI. Eur J Clin Nutr 2010;64:138-45.
10 Hörnell A, Lagström H, Lande B, et al. Protein intake from 0 to 18 years of age and its relation to health: a systematic literature review for the 5th Nordic nutrition recommendations. Food Nutr Res 2013;57:21083

11 Koletzko B, von Kries R, Closa Monasterolo R. Infant feeding and later obesity risk. In: Koletzko B, Desci T, Molnár D, eds. Early nutrition programming and health outcomes in later life. Houten, Netherlands: Springer Netherlands, 2009: 15-29.

12 Rzehak P, Grote V, Lattka E, et al. Associations of IGF-1 gene variants and milk protein intake with IGF-I concentrations in infants at age 6 months - results from a randomized clinical trial. Growth Horm IGF Res 2013;23:149-58.

13 Socha P, Grote V, Gruszfeld D, et al. Milk protein intake, the metabolic-endocrine response, and growth in infancy: data from a randomized clinical trial. Am J Clin Nutr 2011;94:1776S-84.

14 Kirchberg FF, Harder U, Weber M, et al. Dietary protein intake affects amino acid and acylcarnitine metabolism in infants aged 6 months. $J$ Clin Endocrinol Metab 2015;100:149-58.

15 Ketelslegers JM, Maiter D, Maes M, et al. Nutritional regulation of insulin-like growth factor-I. Metabolism 1995;44:50-7.

16 Thissen JP, Ketelslegers JM, Underwood LE. Nutritional regulation of the insulin-like growth factors. Endocr Rev 1994;15:80-101.

17 Hoppe C, Mølgaard C, Dalum C, et al. Differential effects of casein versus whey on fasting plasma levels of insulin, IGF-1 and IGF-1/ IGFBP-3: results from a randomized 7-day supplementation study in prepubertal boys. Eur J Clin Nutr 2009;63:1076-83.

18 Hilbig A, Kersting M. Effects of age and time on energy and macronutrient intake in German infants and young children: results of the Donald study. J Pediatr Gastroenterol Nutr 2006;43:518-24.

19 Damianidi L, Gruszfeld D, Verduci E, et al. Protein intakes and their nutritional sources during the first 2 years of life: secondary data evaluation from the European childhood obesity project. Eur J Clin Nutr 2016;70:1291-7.

20 de Onis M, Onyango AW, Van den Broeck J, et al. Measurement and standardization protocols for anthropometry used in the construction of a new international growth reference. Food Nutr Bull 2004;25:S27-36.

21 Pala V, Reisch LA, Lissner L. Dietary behaviour in children, adolescents and families: the eating habits questionnaire (EHQ). In: Bammann K, Lissner L, Pigeot I, et al, eds. Instruments for health surveys in children and adolescents. Cham: Springer Nature Switzerland, 2019: 103-33.

22 Cole TJ, Bellizzi MC, Flegal KM, et al. Establishing a standard definition for child overweight and obesity worldwide: international survey. BMJ 2000;320:1240-40.

23 Sauzet O, Rehse J, Breiding JH. DistdichoR a R package for the distributional dichotomisation of continuous outcomes. arXiv 2018.

24 Onyango AW, de Onis M, Caroli M, et al. Field-testing the WHO child growth standards in four countries. J Nutr 2007;137:149-52. 